\author{
Military Technical College \\ Kobry El-Kobbah, \\ Cairo, Egypt
}

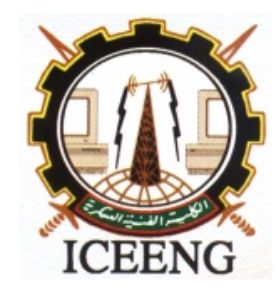

\author{
$8^{\text {th }}$ International Conference \\ on Electrical Engineering \\ ICEENG 2012
}

\title{
A New Single Carrier Frequency Division Multiple Access Based on Discrete Cosine Transform Spread OFDMA.
}

\author{
By \\ Hossam Eldin A. Hassan** Farouk A. K. Al-fuhaidy* Khairy El-barbary**
}

\section{$\underline{\text { Abstract: }}$}

A single carrier frequency division multiple access (SC-FDMA), is a technique that has a similar performance and essentially the same overall complexity as those of orthogonal frequency division multiple access (OFDMA).In this paper, a strong "energy compaction" property of discrete cosine transform (DCT) is exploiting to enhance the performance of SC-FDMA system. A new SC-FDMA system for uplink wireless transmissions is proposed. The proposed system is based on DCT prior to the OFDMA modulation at the transmitter and reverse operation performed at the receiver. The proposed discrete cosine fourier transform (DCFT) SC-FDMA signal is compared with that of the DFT SC-FDMA and OFDMA signals in terms of bit error rate (BER) and peak-to average power ratio (PAPR). Moreover, the proposed system is compared to a recently introduced DCT SC-FDMA system. Simulation results show that the proposed DCFT SC-FDMA provides a significant improvement in BER performance than the DFT SC-FDMA, OFDMA and DCT SC-FDMA. There is a BER improvement of a gain up to $7 \mathrm{~dB}$ compared to OFDMA, $5 \mathrm{~dB}$ when compared to DFT SC-FDMA and $3 \mathrm{~dB}$ when compared to DCT SC-FDMA. In addition, it is found that the PAPR of the DCFT SC-FDMA signals is lower than that of OFDMA signals. Moreover, the proposed system complexity is comparable with the traditional DCT SC-FDMA.

\section{Keywords:}

SC-FDMA, OFDMA, PAPR, DCT and DFT

\footnotetext{
* Egyptian Armed Forces

** Yemani Armed Forces
} 


\section{Introduction:}

Demands for media-rich wireless data services have brought much attention to high speed broadband mobile wireless techniques in recent years. Orthogonal frequency division multiplexing (OFDM), which is a multicarrier communication technique, has become widely accepted. That is due to its robustness against frequency selective fading channels that are common in broadband mobile wireless communications [1]. OFDMA is an extension of OFDM to accommodate multiple simultaneous users. Despite the benefits of OFDM and OFDMA, they suffer a number of drawbacks such as the high PAPR $[2,3,4]$. The high PAPR will cause poor power consumption, inband distortion, and spectrum spreading, when an OFDM signal passes through a nonlinear power amplifier [5]. Single carrier frequency domain equalization (SC-FDE) has recently received more attention as an attractive technology for broadband wireless communications, for its advantages of low PAPR and low sensitivity to carrier frequency offsets, when compared to OFDM $[2,6,7]$. The SCFDMA system is the multiuser counterpart of the SC-FDE system [8]. The main advantages of the SC-FDMA system are that the envelope fluctuations are less pronounced and the power efficiency is higher than that in the conventional OFDMA system [9].

Recently, the traditional DFT SC-FDMA system has been adopted by the third generation partnership project (3GPP) for uplink transmission in the technology standardized for long term evolution of cellular systems [10]. It is introduced in order to keep the PAPR as low as possible. It has similar throughput performance and essentially the same overall complexity as OFDMA. There are two methods of subcarriers mapping in SC-FDMA systems; localized subcarriers mapping which will be referred to as LFDMA, and interleaved subcarriers mapping which will be referred to as IFDMA. In LFDMA, each user uses a set of adjacent subcarriers to transmit his symbols. In IFDMA, the subcarriers used by a user are spread over the entire signal band. More details about the DFT SC-FDMA system are found in [9]. Up to now, the DFT only is used to implement the SCFDMA system. However, it is possible to use other sinusoidal transforms such as the DCT. Recently, DCT based OFDM has received much attention, because it offers certain advantages over the DFT based OFDM [11,12]. The DCT SCFDMA system is recently studied in [13] and provides a good performance enhancement compared to DFT SC-FDMA. More study and addressing of the effect of carrier frequency offsets on DCT SC-FDMA in [14]. The main advantage of the DCT lies in its excellent spectral energy compaction property, which makes most of the samples transmitted close to zero leading to a reduction in the effect of intersymbol interference (ISI). In addition, it uses only real arithmetics rather than the complex arithmetics used in the DFT. This reduces the signal processing complexity, and the inphase/quadrature imbalance $[11,12]$. In this paper, a hybrid DFT and DCT transforms are used to introduce a new system of SC-FDMA. DCT used to transform the modulated 
symbols to frequency domain and IFFT is used to get the time domain signal. Frequency domain equalization (FDE) is used to mitigate the effect of the frequency selective channel. The PAPR and the BER performance of the proposed discrete cosine fourier transform (DCFT) SC-FDMA are studied and compared to DFT SC-FDMA and OFDMA systems. The remainder of this paper is organized as follows. In Section 2, the proposed DCFT SC-FDMA system is presented. In section 3, pulse shaping filters and PAPR are discussed. Section 4 shows the simulation results illustrating the effectiveness of the proposed DCFT SC-FDMA system. Section 5, highlight recently done work is this area of research. Finally, the conclusions and recommendation are given by section 6 .

\section{The Proposed DCFT SC-FDMA System Model:}

The third generation partnership project (3GPP), investigates a modified form of OFDMA for uplink transmissions by the "long-term evolution (LTE)" of cellular systems [11-16] which is called SC-FDMA. In this section the traditional DFT SCFDMA and the proposed DCFT SC-FDMA are presented since the proposed system is not studied in the literature. A schematic block diagram of the DFT/DCFT SC-FDMA system is shown in figure 1. At the transmitter, binary input data passes through one of many possible modulation techniques such as QAM, or M-QAM, introducing modulated symbols. A DFT/DCFT operation performed on each block of $N$ symbols. Then, subcarriers are mapped in the frequency domain using one of three types of mapping, localized, interleaving, or distributed mapping to $M$ subcarriers. The inverse DFT is performed on each block of $M$ subcarriers. After that, a cyclic prefix (CP) of $N c$ symbols is added to the resulting signal. The length of the $\mathrm{CP}$ must be greater than the maximum delay spread of the channel to combat the inter-block interference (IBI) [3]. The signal after DFT can be expressed as follows:

$$
X_{k}=\sum_{n=0}^{N-1} x_{n} e^{-\frac{j 2 \pi}{N} n k}
$$

where $x_{n}$ is an $N \times 1$ vector containing the modulated data symbols. $N$ is a DFT length. The signal after DCT can be expressed as follows:

$X_{k}=\sqrt{\frac{2}{N}} \beta_{k} \sum_{n=0}^{N-1} x_{n} \cos \left(\frac{\pi k(2 n+1)}{2 N}\right)$

where $x_{n}$ is the modulated data symbols, and $\beta_{k}$ given by: 


$$
\beta_{k}=\left\{\begin{array}{c}
\frac{1}{\sqrt{2}}, k=0 \\
1, k=1,2, \ldots, N-1
\end{array}\right.
$$

After the IDFT, the signal can be expressed as follows:

$$
\bar{x}_{m}=\frac{1}{M} \sum_{l=0}^{M-1} \bar{X}_{l} e^{\frac{j 2 \pi}{M} m l}
$$

where $\bar{X}_{l}$ represents the frequency domain samples after subcarrier mapping, $M$ is the IDFT length (number of subcarriers $M>N$ ) and $\bar{x}_{m}$ represents the time symbols after the IDFT. The baseband channel impulse response can, then, be expressed as follows $[7,16]$ :

$$
h(t)=\sum_{l=0}^{L-1} h_{l} \delta\left(t-\tau_{l}\right)
$$

where $h_{l}$ and $\tau_{l}$ are the complex fading and the propogation delay of the $l^{\text {th }}$ path respectively, and $L$ is the number of the multipath components of the channel impulse response $h(t)$.

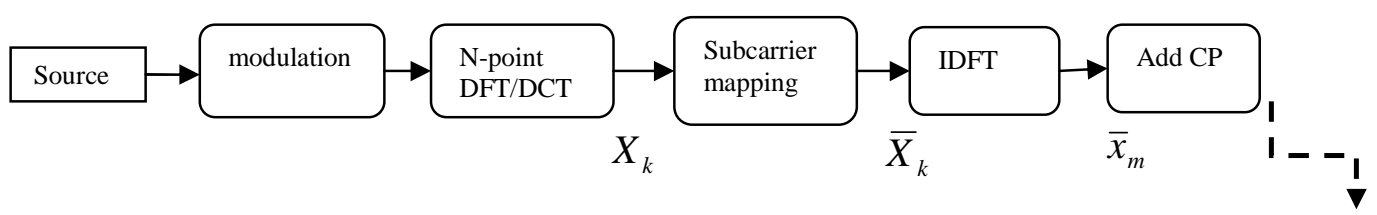

(a) Transmitter of the user $\mathrm{u}$

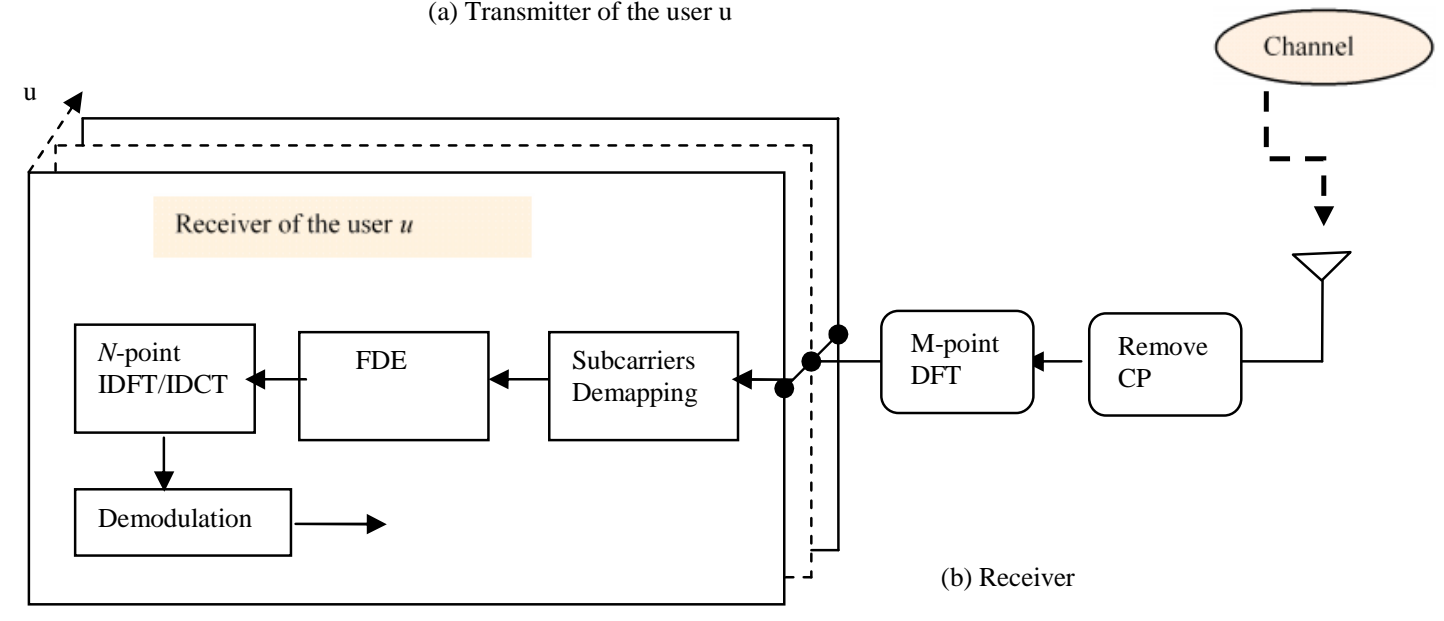

Figure (1): Structure of DFT SC-FDMA/ DCFT SC-FDMA systems over a frequency selective channel. 
At the receiver, the $\mathrm{CP}$ is removed from the received signal. An M-point DFT is performed on the received signal, and getting the frequency domain signal. Then, Frequency Domain Equalization (FDE) and subcarrier demapping are performed. An $N$ point IDFT/IDCT is performed on the resulting signal. Finally, demodulation is performed. After removing $\mathrm{CP}$, the received signal can be expressed as follows:

$r=H \bar{x}+n$

where $\bar{x}$ is an $M \times 1$ vector representing the block of the transmitted symbols and $r$ is an $M \times 1$ vector representing the received symbols. $n$ is an $M \times 1$ vector describing the additive noise. $H$ is an $M \times M$ circulant matrix describing the multipath channel and can be expressed as follows [7]:

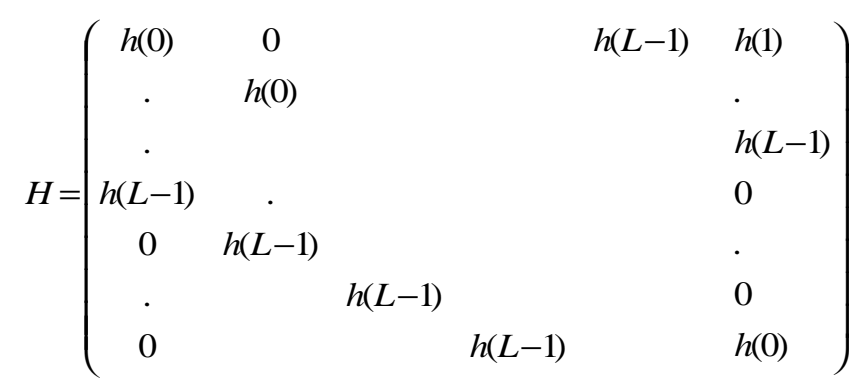

The circulant $H$ matrix can be efficiently diagonalised by the fast fourier transform ( $\Psi$ ) and inverse fast fourier transform $\left(\Psi^{-1}\right)$. It can be written as follows [7]:

$H=\Psi^{-1} \Lambda \Psi$

where $\square$ is an $M \times M$ diagonal matrix containing the fast fourier transform of the circulant sequence of $H$. the FDE complex coefficients $\omega(m)$ can be derived according to the minimum mean square (MMSE) criterion as follows [17]:

$$
\omega(m)=\frac{\Lambda^{*}(m, m)}{|\Lambda(m, m)|^{2}+1 / S N R}
$$

where $S N R$ is the signal-to-noise ratio. The advantage of the frequency domain equalization is its low of computational complexity. But, it requires insertion of $\mathrm{CP}$ which reduces the data rate. 


\subsection{Complexity Evaluation:}

One advantage of the proposed DCFT SC-FDMA system is the lower complexity when compared to the DCT SC-FDMA which proposed in [13] especially at the receiver side. Fig.2 shows the block diagram of the DCT DC-FDMA system. The complexity of the FDE for the DCT SC-FDMA is of $O(M)$ compared to $O(N)$ in DFT SC-FDMA and DCFT SC-FDMA system. Moreover, simulation results show that the proposed DCFT SC-FDMA system is of best performance in terms of BER compared to DCT SCFDMA and DFT SC-FDMA. Thus, the proposed system is of approximately the same complexity as those of the traditional system at the transmitter and the receiver. In addition, if the fast implementation algorithms are taken into consideration, the fast DCT algorithm proposed in [11] can provide fewer computational steps than DFT. This indicates that the complexity of the transmitter and the receiver in the DCFT SC-FDMA system is lower than that in the DFT SC-FDMA system.

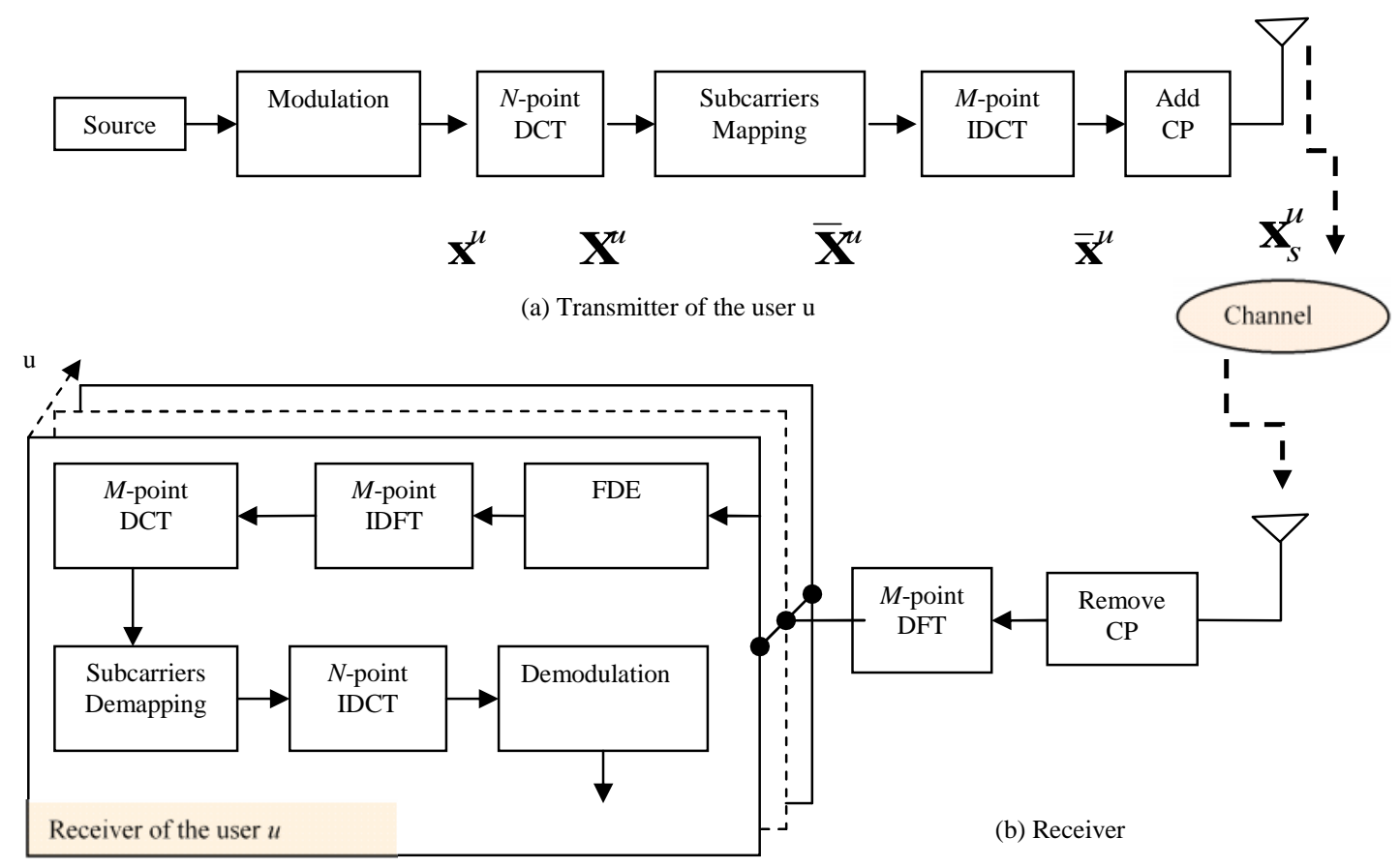

Figure 2: Structure of the DCT SC-FDMA system over a frequency selective channel.

\subsection{Discrete Cosine Transform:}

In DFT, we map the real signal into a complex domain. However, if the input signal has the property of even symmetry, then only cosine series are left. This is the basic idea of 
another important transform, called DCT, which is widely used in many data compression application, such as JPEG and MPEG standards $[12,18]$. On other words, a DCT is a Fourier-related transform similar to the DFT, but using only real numbers.

There are several methods to fold the original sequence in order to get a symmetrical data, which leads to different basic functions of DCT. In this work, we only consider the type-II DCT because of its better energy compaction property. In addition, it is the first one discovered and the most popular in practice [18].

\section{PAPR and Pulse Shaping Filters:}

\subsection{PAPR}

The PAPR is a measure of the peak-to-average power ratio quoted in $\mathrm{dB}$. A positive PAPR in $\mathrm{dB}$ means that we need a power backoff to operate in the linear region of the power amplifier. This reduces the power efficiency of the amplifier and results in a lower mean output power for a given peak power rated device. The PAPR without pulse shaping can be expressed as follows [3, 4]:

$$
P A P R=\frac{P_{p e a k}}{P_{a v}}=\frac{\left.\left.\max _{0 \leq m \leq M-1}\left(\mid \bar{x}_{m}\right)\right|^{2}\right)}{1 / M \sum_{m=0}^{M-1}\left|\bar{x}_{m}\right|^{2}} \quad m=0,1, \ldots, M+N_{c}-1 .
$$

where $\bar{x}_{\mathrm{m}}$ is the transmitted signal after the cyclic prefix.

\subsection{Pulse Shaping Filters}

To evaluate the effect of the pulse shaping filter on the HT SC-FDMA system, two pulse shaping filters are considered in this paper, the raised cosine (RC) filter and the root raised cosine (RRC) filter. The impulse response of the raised cosine filter is given by [19],

$h(t)=\operatorname{sinc}\left(\pi \frac{t}{T}\right) \frac{\cos \left(\frac{\pi a t}{T}\right)}{1-\frac{4 \alpha^{2} t^{2}}{T^{2}}}$

where $\alpha$ is the roll-off factor, which lies between 0 and 1 , and $T$ is the symbol-period. The root raised-cosine filter is an implementation of a low-pass Nyquist filter, which has the property of vestigial symmetry. The impulse response of such a filter is given by [19]: 
$h(t)=\frac{\sin \left(\pi \frac{t}{T}(1-\alpha)\right)+4 \alpha \frac{t}{T} \cos \left(\pi \frac{t}{T}(1+\alpha)\right)}{\pi \frac{t}{T}\left(1-\left(4 \alpha \frac{t}{T}\right)^{2}\right)}$

\section{Simulation Results}

In this section, the performance of the proposed DCFT SC-FDMA system, using perfect channel knowledge, is evaluated by simulations. The Monte Carlo simulation method is used. For the comparison purpose, the DFT SC-FDMA and the OFDMA systems are also simulated. The simulation parameters are tabulated in Table 1.

Table.1. Simulation Parameters

\begin{tabular}{|l|l|l|}
\hline & Description & Parameters \\
\hline \multirow{4}{*}{ Transmitter } & System bandwidth & 5 MHz \\
\cline { 2 - 3 } & Modulation & QPSK and 16QAM \\
\cline { 2 - 3 } & CP & 20 samples \\
\cline { 2 - 3 } & Transmitter IDFT size & $\mathrm{M}=256$ \\
\cline { 2 - 3 } & Subcarrier spacing & $9.765625 \mathrm{kHz}$ \\
\cline { 2 - 3 } & $\begin{array}{l}\text { SC-FDMA input block } \\
\text { size }\end{array}$ & 64 symbols \\
\cline { 2 - 3 } & Subcarrier mapping & Localized and Interleaved \\
\hline Channel & Channel model & $\begin{array}{l}\text { Vehicular A outdoor } \\
\text { channel }\end{array}$ \\
\cline { 2 - 3 } & Noise environment & AWGN \\
\hline Receiver & Channel estimation & Perfect channel knowledge \\
\cline { 2 - 3 } & Equalization & MMSE \\
\hline & No. of runs & $10^{\wedge} 4$ \\
\hline
\end{tabular}

The convolutional code with memory length 7 and octal generator polynomial $(133,171)$ is used. QPSK and 16-QAM are used. The channel model used for simulations is the vehicular A outdoor channel [20]. It has six Rayleigh fading taps at delays of 0, 310, $710,1090,1730$ and $2510 \mathrm{~ns}$, with relative powers of $0 \mathrm{~dB},-1 \mathrm{~dB},-9 \mathrm{~dB},-10 \mathrm{~dB},-15$ $\mathrm{dB}$ and $-20 \mathrm{~dB}$, respectively. The vehicular A channel has a mobile speed of $120 \mathrm{Km}$ per hour, which corresponds to a Doppler spread of $223 \mathrm{~Hz}$ for a carrier frequency of 2 GHz.

\subsection{BER Performance}

Fig.3 illustrates the BER performance of the DCFT SC-FDMA system compared to the DFT SC-FDMA and the OFDMA systems, with different subcarriers mapping methods, 
localized and interleaved, and different modulation formats over the vehicular A channel. $N=64, M=256$, and $Q=4$ users are used. It can be observed that DCFT SCFDMA system provides a significant BER performance improvement over the DFT SCFDMA and the OFDMA systems. For example, for QPSK modulation, at a BER $=10^{-3}$, the performance gain is about $4 \mathrm{~dB}$ for DCFT LFDMA, and about $5 \mathrm{~dB}$ for DCFT IFDMA, when it is compared to that of the DFT LFDMA and the DFT IFDMA, respectively. For 16QAM, the performance of the DCFT SC-FDMA system is also better than that of the DFT-SC-FDMA and the OFDMA systems. The performance of the DFT-SC-FDMA system is worse than that of the OFDMA and the DCFT-SCFDMA systems for 16-QAM.

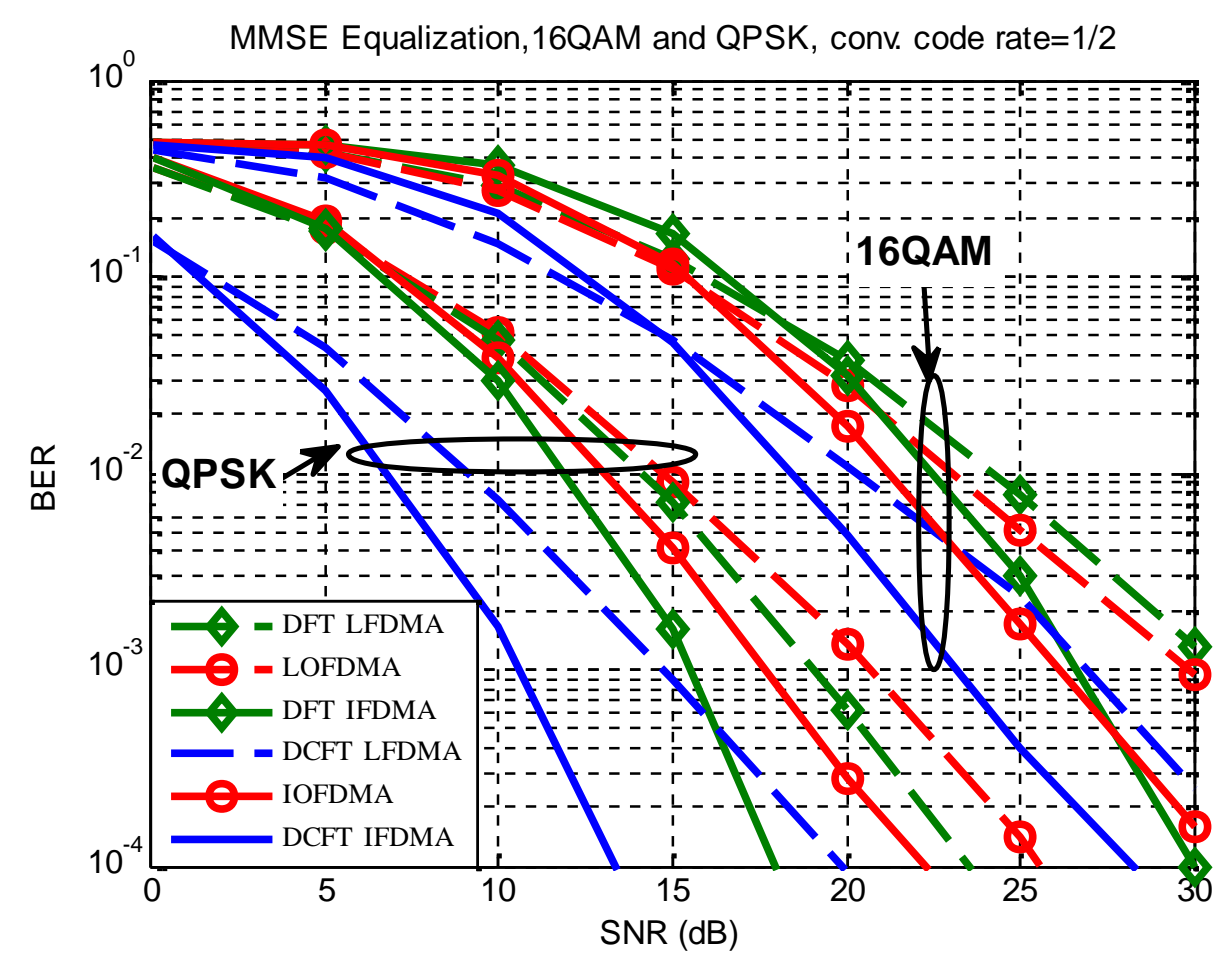

Figure (3): BER vs. SNR of the proposed DCFT SC-FDMA compared to DFT SC-FDMA and OFDMA systems.

At a BER $=10^{-3}$, the SNR improvements for the DCFT SC-FDMA system as compared to the DFT SC-FDMA and the OFDMA systems are tabulated in Table 2.

It is clear from the previous figure that the proposed DCFT SC-FDMA system has a significant BER performance enhancement when it compared to the traditional DFT SCFDMA system and OFDMA system when localized or interleaved subcarrier mapping are used. 
Proceedings of the $\boldsymbol{8}^{\text {th }}$ ICEENG Conference, 29-31 May, 2012

Table (2): SNR improvement of the proposed DCFT SC-FDMA at a BER $=10^{-3}$

\begin{tabular}{|l|l|l|l|l|}
\hline \multirow{2}{*}{$\begin{array}{l}\text { The proposed } \\
\text { system }\end{array}$} & \multicolumn{2}{|c|}{$\begin{array}{c}\text { SNR improvement as } \\
\text { compared to the } \\
\text { DFT SC-FDMA }\end{array}$} & \multicolumn{2}{c|}{$\begin{array}{l}\text { SNR improvement as compared } \\
\text { to the OFDMA }\end{array}$} \\
\cline { 2 - 6 } & QPSK & $16 \mathrm{QAM}$ & QPSK & $16 \mathrm{QAM}$ \\
\hline DCFT LFDMA & $4 \mathrm{~dB}$ & $3 \mathrm{~dB}$ & $5.5 \mathrm{~dB}$ & $2.5 \mathrm{~dB}$ \\
\hline DCFT IFDMA & $5 \mathrm{~dB}$ & $3.25 \mathrm{~dB}$ & $7 \mathrm{~dB}$ & $3 \mathrm{~dB}$ \\
\hline
\end{tabular}

Fig.4 shows simulation of the proposed system compared to the DCT SC-FDMA system in terms of BER against SNR.

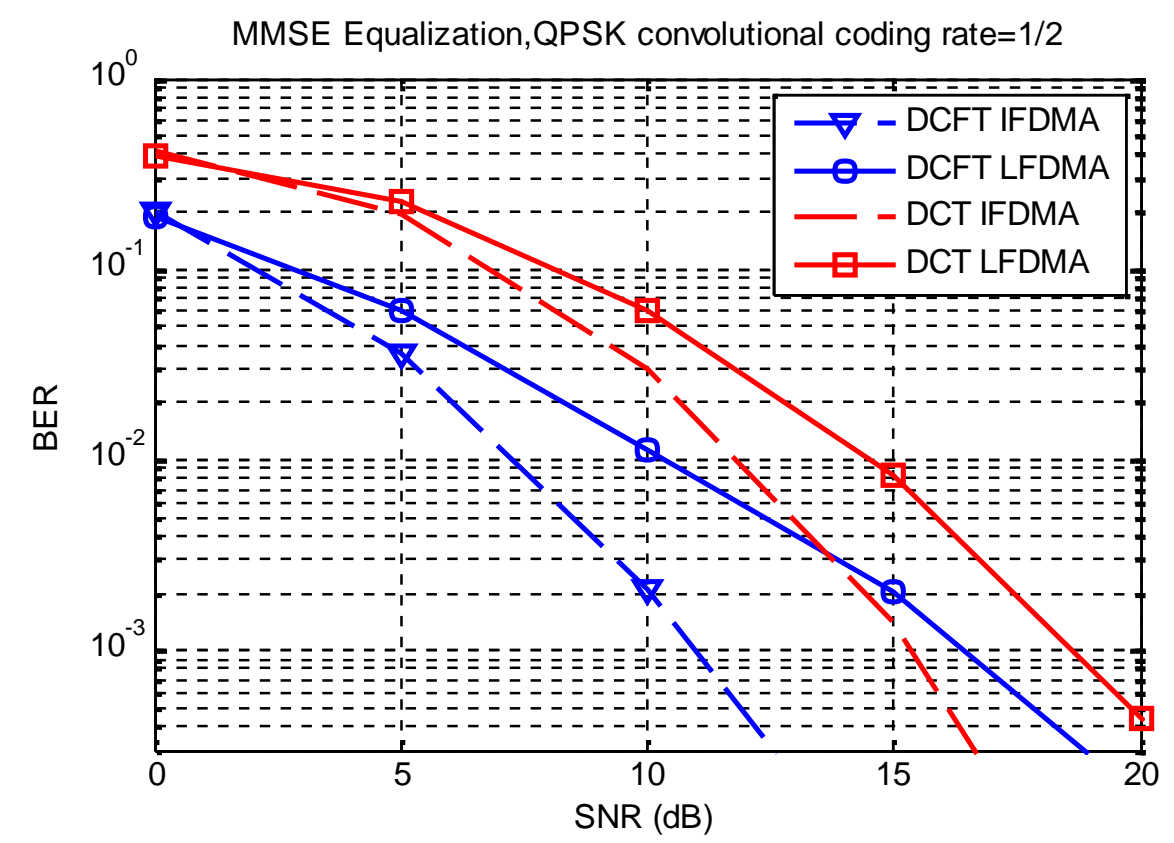

Figure (4): BER vs. SNR of the proposed DCFT SC-FDMA compared to DCT SC-FDMA, QPSK modulation.

It is shown from previous figure the proposed scheme has a significant BER enhancement compared to the DCT SC-FDMA. At BER=0.001, the SNR improvement of DCFT IFDMA compared to DCT IFDMA is $4 \mathrm{~dB}$ and of $2 \mathrm{~dB}$ for DCFT LFDMA compared to DCT LFDMA.

\subsection{PAPR Simulation Results}

The complementary cumulative distribution function (CCDF) of the PAPR, which is the probability that PAPR is higher than a certain value, is calculated by Monte Carlo simulations. The CCDF of PAPR for DCFT SC-FDMA, DFT SC-FDMA, and OFDMA 
are evaluated and compared with different pulse shaping filters and modulation formats. No pulse shaping is applied in the case OFDMA. In figure 5, plots of CCDF of the PAPR for DCFT IFDMA, DCFT LFDMA, and OFDMA are shown with different modulation formats. It is clearly seen that, without pulse shaping, the DCFT IFDMA has lower PAPR than the OFDMA by about $3.75 \mathrm{~dB}$ for QPSK and by about $2.25 \mathrm{~dB}$ for 16-QAM, while PAPR of DCFT LFDMA is lower than that of OFDMA by about 3 $\mathrm{dB}$ for QPSK and by about $2 \mathrm{~dB}$ for 16-QAM. With pulse shaping, raised-cosine or root raised-cosine, with roll-off factor of 0.22 , it can be seen that the PAPR increases by about $1 \mathrm{~dB}$ for DCFT IFDMA, whereas the PAPR of the DCFT LFDMA increases by about $0.25 \mathrm{~dB}$ for QPSK and $0.5 \mathrm{~dB}$ for 16-QAM. It is also noted that the PAPR of the DCFT SC-FDMA system depends on the modulation format used. A full comparison of PAPR for different modulation formats at a $\mathrm{CCDF}=10^{-3}$ is approximately given in Table 3.

Table (3): PAPR for DCFT IFDMA, DCFT LFDMA and OFDMA at CCDF $=10^{-3}$.

\begin{tabular}{|l|l|l|l|l|}
\hline Modulation & Pulse shaping & DCFT IFDMA & $\begin{array}{l}\text { DCFT } \\
\text { LFDMA }\end{array}$ & OFDMA \\
\hline \hline \multirow{4}{*}{ QPSK } & None & $7.4 \mathrm{~dB}$ & $7.60 \mathrm{~dB}$ & $10.75 \mathrm{~dB}$ \\
\cline { 2 - 5 } & RC & $8.5 \mathrm{~dB}$ & $7.75 \mathrm{~dB}$ & N/A \\
\cline { 2 - 5 } & RRC & $8.25 \mathrm{~dB}$ & $7.75 \mathrm{~dB}$ & N/A \\
\hline \multirow{3}{*}{ 6QAM } & None & $8.25 \mathrm{~dB}$ & $8.75 \mathrm{~dB}$ & $10.75 \mathrm{~dB}$ \\
\cline { 2 - 5 } & RC & $9.25 \mathrm{~dB}$ & $9 \mathrm{~dB}$ & N/A \\
\cline { 2 - 5 } & RRC & $9.25 \mathrm{~dB}$ & $9.25 \mathrm{~dB}$ & N/A \\
\hline
\end{tabular}

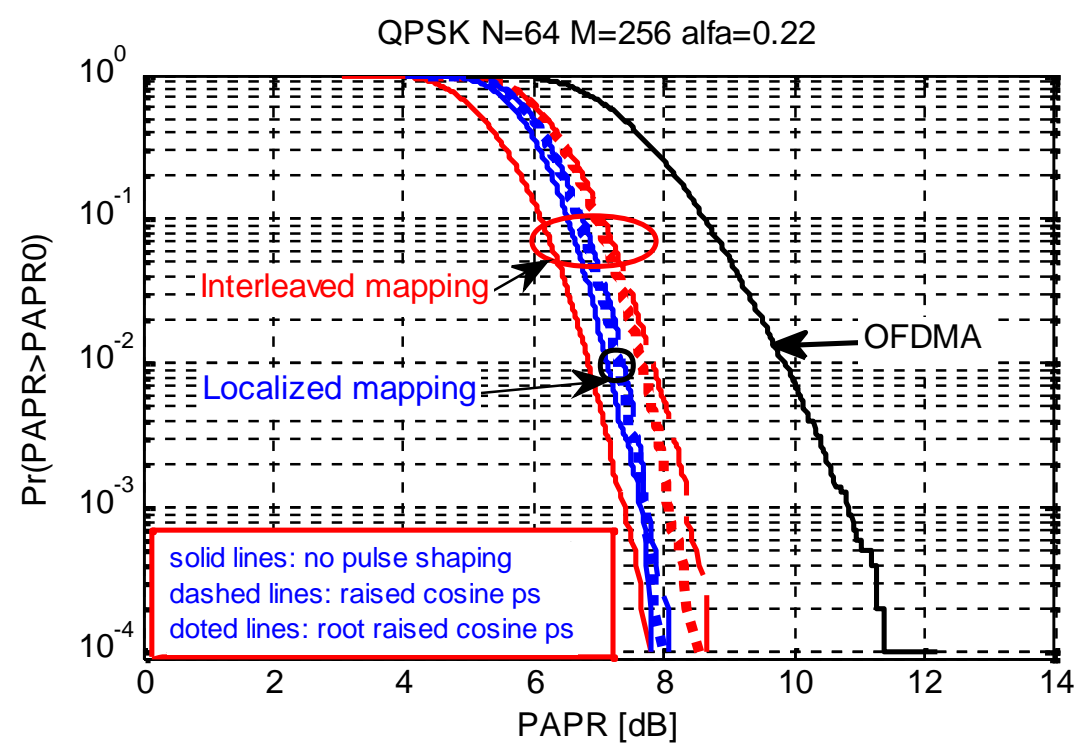

(a) 


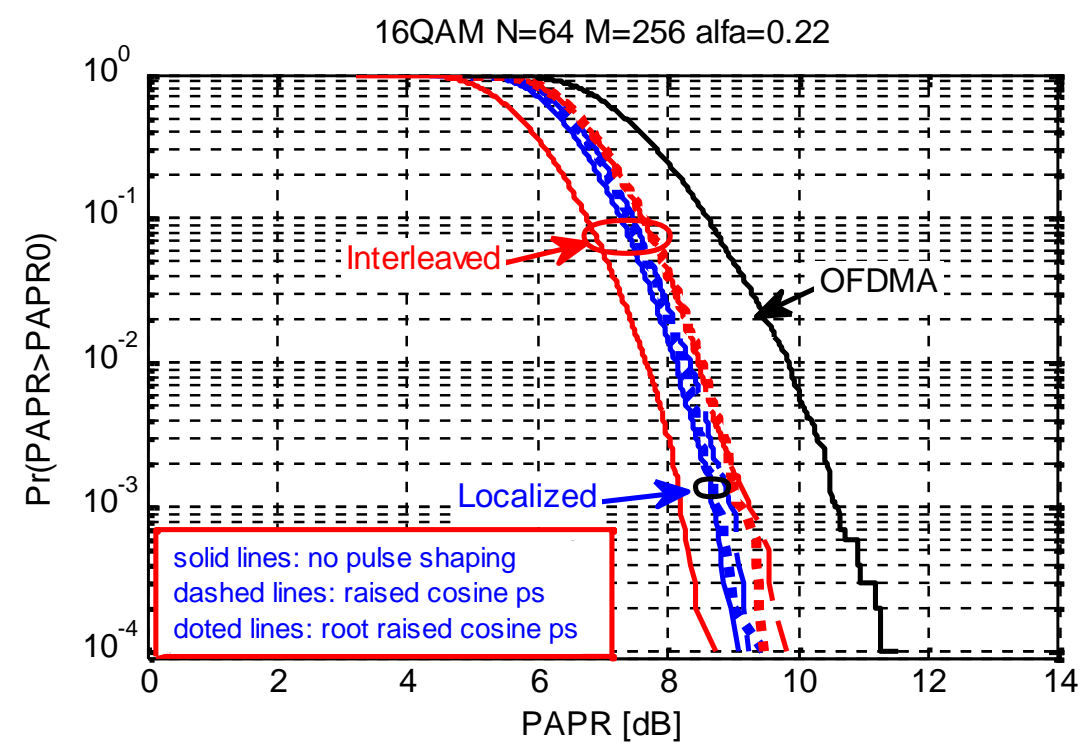

(b)

Figure (5): comparison of CCDF of PAPR for DCFT IFDMA, DCFT LFDMA and OFDMA. (a) QPSK. (b) 16QAM

Fig.6 studies and shows the CCDF of PAPR for the DFT IFDMA, DFT LFDMA and OFDMA systems with different modulation formats and different pulse shaping filters.

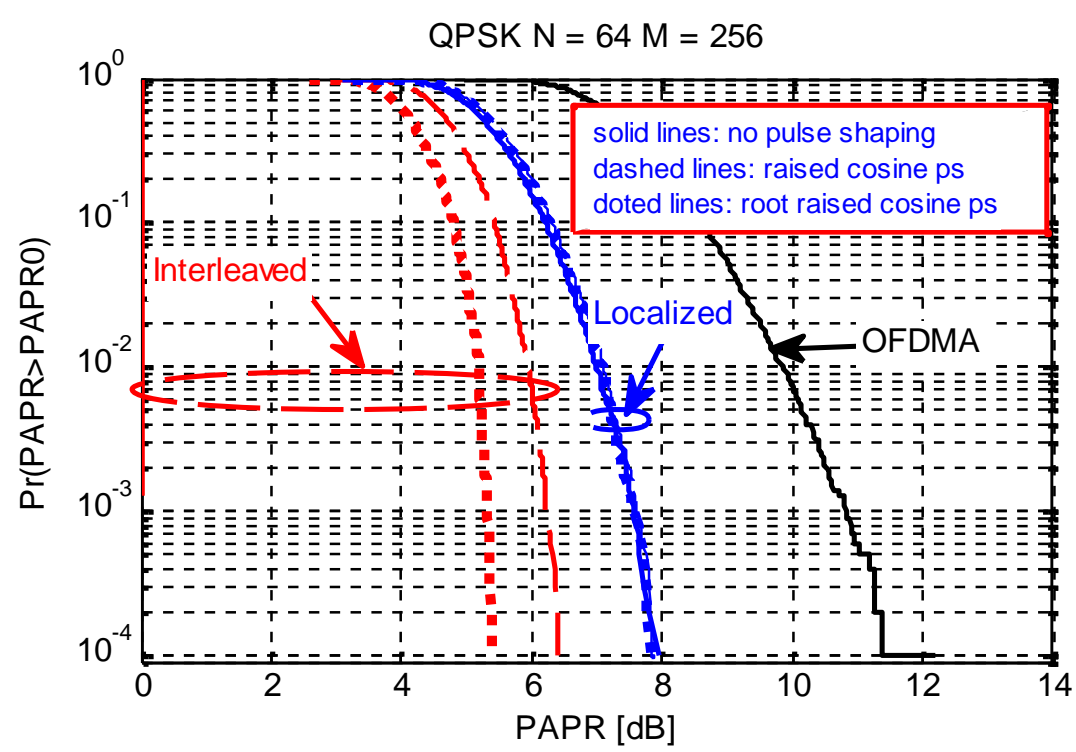

(a) 


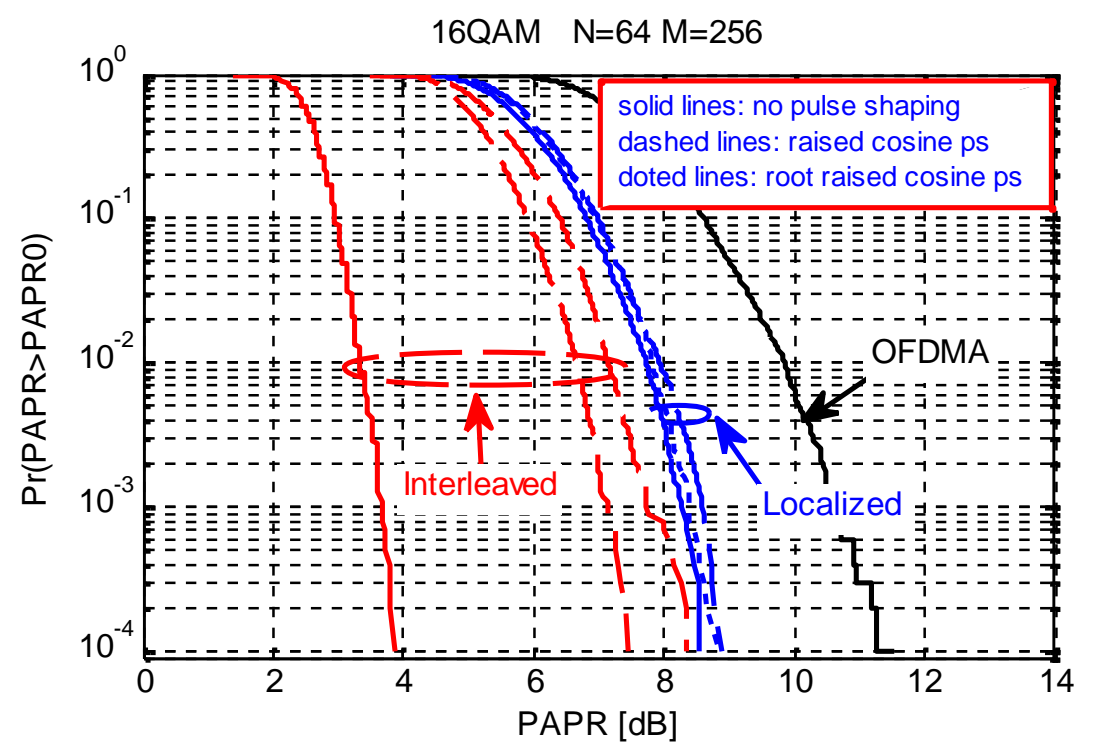

(b)

Figure (6): comparison of CCDF of PAPR for DFT IFDMA, DFT LFDMA and OFDMA. (a) QPSK. (b) 16QAM

From the previous figures, it is shown that for localized mapping with different modulation format and with or without pulse shaping, the DCFT SC-FDMA and DFT SC-FDMA have similar PAPR. Whereas, the DFT IFDMA has lower PAPR compared to DCFT IFDMA. But, they are of lower PAPR than the OFDMA system.

\section{5. $\underline{\text { Conclusions }}$}

In this paper, a new transceiver scheme of the DFT SC-FDMA was studied. The proposed system is based on, DCT spread OFDMA rather than DFT spread OFDMA. The proposed scheme was verified using simulation that it has a significant performance enhancement over DFT SC-FDMA and OFDMA in terms of BER with a gain factor approaches $5 \mathrm{~dB}$ with respect to DFT SC-FDMA and $7 \mathrm{~dB}$ when compared to OFDMA with QPSK modulation. In addition, the proposed DCFT SC-FDMA system has best BER than the recently suggested DCT SC-FDMA in [13] and lower complexity especially at the receiver side. Moreover, the proposed DCFT SC-FDMA has lower complexity than DFT SC-FDMA when using fast implementation of DCT. The tradeoff of the proposed system is the higher PAPR of interleaved mapping when compared to DFT IFDMA. But, it also provides lower PAPR compared to OFDMA.

\section{6. $\underline{\text { References }}$}

[1] L J Cimini, Jr, Analysis and Simulation of a Digital Mobile Channel Using Orthogonal Frequency Division Multiplexing, IEEE Trans. Communication., vol. 33, no. 7, July 1985, pp. 665-675. 
[2] D Falconer, S L Ariyavisitakul, A Benyamin-Seeyar, and B Eidson B, Frequency domain equalization for single-carrier broadband wireless systems, IEEE Commun. Mag., 2002, 40, pp. 58-66.

[3] H G Myung, J Lim and D J Goodman, Single Carrier FDMA for Uplink Wireless transmission, Proceeding of the IEEE Vehicular Technology Magazine. , vol. 1, no. 3, Sep. 2006.

[4] S H Han and J H Lee, An Overview of Peak-to-Average Power Ratio Reduction Techniques for Multicarrier Transmission, IEEE Wireless Communications, vol. 12, no. 2, 2005, pp. 56-65.

[5] H Schulze and C Luders, Theory and applications of OFDM and CDMA, Wiley, 2005.

[6] Y Wang, X Dong, P H Wittke, and M Shaomin, Cyclic prefixed single carrier transmission in ultra-wideband communications, IEEE Trans. wireless Communs. , vol. 5, no. 8, pp. 2017 - 2021, Aug 2005.

[7] F S Al-kamali, M I Dessouky, B M Sallam and F E El-Samie, Performance evaluation of cyclic prefix CDMA systems with frequency domain interference cancellation, Digital Signal Processing Journal, Elsevier Inc, vol. 19, no. 1, pp. 2-13, Jan. 2009.

[8] F S Al-kamali, M I Dessouky, B M Sallam, and F E El-Samie and F. Shawki, A new transceiver scheme for SC-FDMA implementing the wavelet transform and the PAPR reduction methods, IET Communication, vol. 4, no. 1, pp. 69-79, Jan. 2010..

[9] H G Myung, and D J Goodman, Single carrier FDMA a new air interface for long term evaluation, John Wiley \& Sons, Ltd., 2008.

[10] 3rd Generation Partnership Project (3GPP), Technical specification group radio access network, Physical layer aspects for evolved UTRA (Release 7).

[11] G D Mandyam, Sinusoidal transforms in OFDMA system, IEEE Trans. On Broadcasting. vol. 50, no. 2, June 2004.

[12] P. Tan and N. C. Beaulieu, A Comparison of DCT-Based OFDM and DFT-Based OFDM in Frequency Offset and Fading Channels, IEEE Trans. On Comm., vol. 54, NO. 11, Nov. 2006.

[13] F S Al-kamali, M I Dessouky, B M Sallam, F E Abd El-Samie, and F Shawki, A New Single Carrier FDMA System Based on the Discrete Cosine Transform, in Proc. of the ICCES'9 Conf., Cairo, Egypt, pp. 555-560, 14-16 Dec. 2009.

[14] F S Al-kamali, M I Dessouky, B M Sallam, F E Abd El-Samie, and F Shawki, Carrier Frequency Offset Problem in DCT-SC-FDMA System: Investigation and Compensation, ISRN Commun. and Networking Journal, Hindawi Cor. vol 2011, pp. 1-7, 2011.

[15]. H Ekström, A Furuskär, J Karlsson, M Meyer, S Parkvall, J Torsner, and M Wahlqvist, Technical Solutions for the $3 G$ Long-Term Evolution, IEEE Commun. Mag., vol. 44, no. 3, pp. 38-45, Mar. 2006. 
[16] F Adachi, D Garge, S Takaoka and K Takeda Broadband CDMA techniques, IEEE Wirel. Comm, 2005, 12, (2), pp. 8-18.

[17] Y C Liang, Block-iterative GDFE (BI-GDFE) for CPCDMA and MC-CDMA, Proc. IEEE VTC, 2005, 5, (6), pp. 3033-3037.

[18] Y. Han and J. Leou, Detection and Correction of Transmission Errors in JPEG Images, IEEE Trans. On Circuits and Systems for video technology, vol. 8, no. 2, April 1998, pp. 221-231.

[19] T S Rappaport, Wireless communications: principles and practice, Second Edition. Prentice Hall, 2002.

[20] Alpha Concept Group: Wideband direct sequence CDMA (WCDMA) evaluation document (3.0), Tdoc SMG 905/97, Madrid, Spain, December 1997. 